\title{
Surfactant Protein D for Pathological Evaluation of Infant Acute Respiratory Distress Syndrome Caused by Respiratory Syncytial Virus Infection
}

\author{
Daisuke Tamura, Shun Inoue, Takatoshi Oishi, Ayafumi Ozaki, Takanori Yamagata \\ Department of Pediatrics, Jichi Medical University, Tochigi, Japan \\ Email: dtamura@jichi.ac.jp
}

How to cite this paper: Tamura, D., Inoue, S., Oishi, T., Ozaki, A. and Yamagata, T. (2020) Surfactant Protein D for Pathological Evaluation of Infant Acute Respiratory Distress Syndrome Caused by Respiratory Syncytial Virus Infection. Open Journal of Pediatrics, 10, 652-660.

https://doi.org/10.4236/ojped.2020.104067

Received: October 19, 2020

Accepted: December 1, 2020

Published: December 4, 2020

Copyright $\odot 2020$ by author(s) and Scientific Research Publishing Inc. This work is licensed under the Creative Commons Attribution International License (CC BY 4.0).

http://creativecommons.org/licenses/by/4.0/

(c) (i) Open Access

\begin{abstract}
Pediatric respiratory syncytial viral infection (RS) usually shows relatively good outcome; however, when it accompanies acute respiratory distress syndrome (ARDS), this becomes fatal. We experienced three pediatric patients with RS + ARDS, with all showing good outcome with steroid pulse therapy. We wish to emphasize; 1) steroid pulse therapy may become an option for this condition, and 2) plasma KL-6 and surfactant protein D levels may become a biomarker reflecting the disease progression/condition. Patients were, aged 1 month, 1 year 5 months, and 1 year 11 months. In all three, the respiratory condition deteriorated rapidly, requiring invasive ventilator management. Although the effectiveness of steroid treatment for ARDS is controversial, very severe condition prompted us to employ steroid pulse therapy, after which, oxygenation rapidly improved without adverse events. Plasma KL-6 and surfactant protein D levels were measured during exacerbations of ARDS, steroid pulse therapy, and recovery. Surfactant protein D levels were closely associated with oxygenation, suggesting this substance level might be a biomarker of ARDS caused by the disruption of the alveolar epithelial lining and to understand oxygenation without time lag.
\end{abstract}

\section{Keywords}

Infants, Respiratory Syncytial Virus, Acute Respiratory Distress Syndrome, Surfactant Protein D, KL-6

Respiratory syncytial viral infection (RS) is the major cause of lower respiratory tract illness in children [1] [2]. In infants less than 1 year of age and with lower 
respiratory infection, up to $80 \%$ are due to RS. For most children, an initial RS normally occurred within the first 2 years of life. Clinical manifestations range from mild upper respiratory tract infections to severe lower respiratory tract disorders such as bronchiolitis and/or pneumonia. Well-defined high-risk groups, including infants with prematurity, pre-existing lung disease, congenital heart disease, and immunodeficiencies are more likely to show a prolonged and severe course of RS [3] [4] [5]. Although some infants need assisted ventilation due to recurrent apnea or respiratory failure, the clinical course of RS is generally good, and some previous studies have shown low mortality rates of $2 \%-5 \%$, even for high-risk group patients [6] [7] [8] [9].

Acute respiratory distress syndrome (ARDS) secondary to RS has been described in some infants previously [10] [11] [12]. The disease entity defined as ARDS is an acute and severe parenchymal lung injury characterized by hypoxia, diffuse radiographic infiltrates, decreased pulmonary compliance, and pulmonary edema [13]. The diversity of patients identified as having ARDS makes it difficult for epidemiological and clinical studies to evaluate mortality rates and risk factors, or to measure the benefit of therapeutic interventions including steroid therapy [14] [15]. In contrast to the overall low mortality rates reported for infants with RS, published data report the mortality rate in pediatric patients with ARDS to be in excess of 24\% [16].

We experienced three infants of RS and developed severe ARDS. Steroid pulse therapy was selected for the treatment of severe ARDS and the clinical course was favorable. The aim of this case report is to show the effectiveness of steroid pulse therapy and the usefulness of plasma surfactant protein D level (SP-D), a serum marker that rapidly and accurately reflects changes in oxygenation in infants with severe ARDS due to RS.

Case 1 was a 1-month-old girl. She had a nasal discharge from 4 days before admission, cough with dyspnea, and poor feeding that worsened days before admission. She was hospitalized with acute bronchopneumonia with hyper-infiltration and restricted regional atelectasis on the lower lobe of the right lung by chest X-ray. She showed retractive breathing, and crackles and wheezing were enhanced in all chest compartments. Vital signs were as follows: body temperature $38.4^{\circ} \mathrm{C}$, respiratory rate $32 / \mathrm{min}$, and $\mathrm{SpO}_{2} 90 \%$ in room air. Laboratory studies demonstrated elevated white blood cells (WBC) $19,100 / \mu \mathrm{L}$, C-reactive protein (CRP) $4.1 \mathrm{mg} / \mathrm{L}$, and lactose dehydrogenase (LDH) $506 \mathrm{U} / \mathrm{L}$. T/B and NK cell analysis: CD4 37.8\%, CD8 31.2\%, CD19 15.0\%, and CD56 11.2\%. IgG/A/M levels were 993, 294, and $219 \mathrm{mg} / \mathrm{dL}$, respectively. Chest computed tomography (CT) showed consolidation with air Broncho gram observed on both dorsal lungs. In addition, granular or patchy shadows were scattered, and thickening of bronchial bundles was recognized as cord-like shadows in the lower lobes of both sides. Invasive mechanical ventilator management was started the day after admission. The partial pressure of arterial oxygen $\left(\mathrm{PaO}_{2}\right)$ /fraction of inspiratory oxygenation $\left(\mathrm{FiO}_{2}\right)$ ratio was 142 , and $\mathrm{KL}-6$ and SP-D levels were $1304 \mathrm{U} / \mathrm{mL}$ and $118 \mathrm{ng} / \mathrm{ml}$, respectively (Table 1 ). On day 9 
Table 1. Changes in KL-6 levels and surfactant protein D levels before and after steroid pulse therapy. KL-6 levels and surfactant protein D levels were associated with the partial pressure of arterial oxygen/fraction of inspiratory oxygenation ratio in all patients before and after steroid pulse therapy. Surfactant protein D levels changed rapidly in parallel with the partial pressure of arterial oxygen/fraction of inspiratory oxygenation ratio; however, KL-6 changes were slightly delayed in all cases. At discharge, surfactant protein $\mathrm{D}$ levels in all cases had recovered to normal although KL-6 levels were still high.

\begin{tabular}{cccccccccc}
\hline Case 1 & & & & & & & & & \\
\hline Days after onset & 5 & $6^{*}$ & $9^{\dagger}$ & 10 & $12^{\ddagger}$ & 14 & $19^{\S}$ & 33 & 99 \\
KL-6 (U/ml) & 1187 & 1304 & 1475 & NA & 1533 & NA & 864 & 396 & 189 \\
SP-D (ng/ml) & NA & 118 & 177 & NA & 129 & NA & 41.5 & NA & NA \\
P/F ratio & 178 & 142 & 122 & 138 & 250 & NA & NA & NA & NA \\
Case 2 & & & & & & & & & \\
Days after onset & $5^{*}$ & $6^{\dagger}$ & 7 & $11^{\ddagger}$ & 13 & $19^{\S}$ & 38 & 64 & 78 \\
KL-6 (U/ml) & 1651 & 2003 & 2890 & 5557 & 5282 & NA & 1732 & 1191 & 507 \\
SP-D (ng/ml) & 121 & 212 & 411 & 377 & 291 & NA & 94.7 & NA & 56.1 \\
P/F ratio & 182 & 112 & 95 & 183 & 255 & NA & NA & NA & NA \\
Case 3 & & & & & & & & & \\
Days after onset & 5 & $6^{*}$ & $7^{\dagger}$ & 10 & $15^{\dagger}$ & 17 & $20^{\ddagger}$ & $32^{\S}$ & 59 \\
KL-6 (U/ml) & 296 & 1396 & 2889 & 3763 & 7074 & 6870 & 4204 & 2218 & 684 \\
SP-D (ng/ml) & NA & 122 & 451 & 753 & 626 & 472 & 145 & 105 & 81.9 \\
P/F ratio & NA & 240 & 156 & 77 & 205 & 229 & 287 & NA & NA \\
\hline
\end{tabular}

${ }^{*}$ Invasive mechanical ventilation; ${ }^{\dagger}$ Steroid Pulse Therapy; ${ }^{\ddagger}$ Extubation and non-invasive ventilation; ${ }^{\S}$ Discharge; NA: not applicable.

after onset, KL-6 and SP-D levels increased to $1475 \mathrm{U} / \mathrm{mL}$ and $177 \mathrm{ng} / \mathrm{ml}$, and the $\mathrm{PaO}_{2} / \mathrm{FiO}_{2}$ ratio was reduced to 122 . Steroid pulse therapy was started on day 9 after onset.

Case 2 was a 1-year 11-month-old girl with Dandy-Walker syndrome. She was admitted to hospital on day 4 after onset with worsening severe cough, and a loss of appetite for 3 days. Vital signs were as follows: body temperature $37.6^{\circ} \mathrm{C}$, respiratory rate $28 / \mathrm{min}$, and $\mathrm{SpO}_{2} 89 \%$ in room air. Laboratory studies demon-

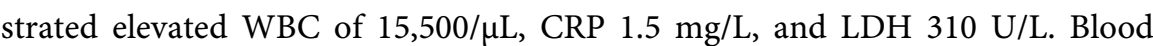
tests for immune system components at a previous hospitalization did not show any abnormalities. The amount of IgG was age-appropriate. Because respiratory conditions including retractive breathing gradually worsened, non-invasive ventilator management with a high-flow nasal cannula was started on the day of admission. The day after admission, her respiratory rate was $32 / \mathrm{min}$ and there was less wheezing and crackling in the lower chest compared with that at admission. Chest CT showed a large air space along the bottom of the lungs, the mediastinum, and the hilum. An area of increased concentration centered on consolidation with the air Broncho gram spread segmentally into the dorsal part of both lungs. In addition, light ground-glass opacity was diffuse as seen from the 
hilum (Figure 1). Due to the rapid progressive deterioration of respiratory conditions, invasive mechanical ventilator management was started. Six days after onset, the $\mathrm{PaO}_{2} / \mathrm{FiO}_{2}$ ratio rapidly decreased to 112 , and KL-6 and SP-D levels were markedly increased at $2003 \mathrm{U} / \mathrm{mL}$ and $212 \mathrm{ng} / \mathrm{ml}$, respectively (Table 1). Steroid pulse therapy was started on day 6 after onset.

Case 3 was a 1-year 5-month-old girl with a vertebral defect, anal atresia, cardiac malformations, tracheoesophageal fistula with esophageal atresia, radial dysplasia, renal dysplasia, and limb anomaly (VACTERL association). She had a dry cough for 4 days. Five days after onset, her respiratory condition gradually worsened, and she was hospitalized because of dyspnea. Vital signs were as follows: body temperature $38.1^{\circ} \mathrm{C}$, respiratory rate $32 / \mathrm{min}$, and $\mathrm{SpO}_{2} 88 \%$ in room air. Laboratory studies demonstrated WBC $8900 / \mu \mathrm{L}, \mathrm{CRP}<0.1 \mathrm{mg} / \mathrm{L}$, and KL-6 296 U/mL. T/B and NK cell analysis: CD4 35.0\%, CD8 22.3\%, CD19 15.1\%, and CD56 34.0\%. IgG/A/M levels were 582, 42, and $97 \mathrm{mg} / \mathrm{dL}$, respectively. Chest CT showed ground-glass shadows or shadows with Broncho gram dominating the dorsal predominance of both lungs. Lung field consolidation indicated strong inflammation (Figure 2). Six days after onset, retractive breathing had worsened, and invasive mechanical ventilation management was started. Seven days after onset, her $\mathrm{PaO}_{2} / \mathrm{FiO}_{2}$ ratio had reduced to 156, wheezes were heard in all chest compartments, and KL-6 and SP-D levels were elevated to $2889 \mathrm{U} / \mathrm{mL}$ and $451 \mathrm{ng} / \mathrm{ml}$, respectively (Table 1 ). Steroid pulse therapy started on day 7 after onset.

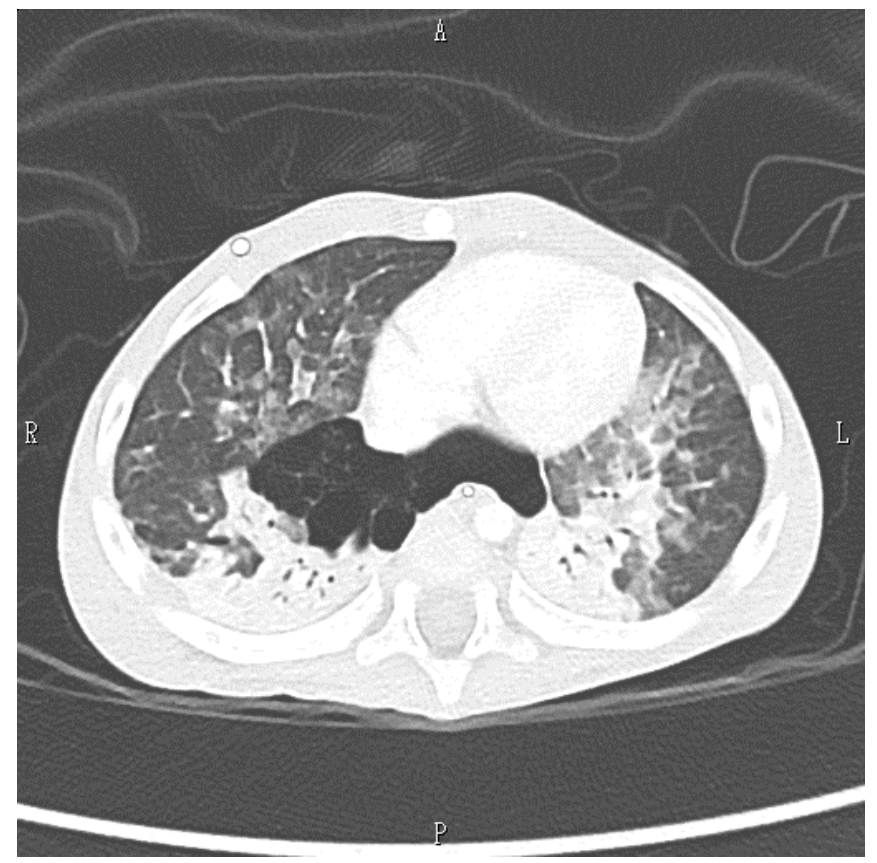

Figure 1. Case 2, Chest CT image on day 4 after onset. A large air space is observed along the bottom of the lungs, the mediastinum of the mediastinum, and the hilum. An area of increased concentration centered on consolidation with air bronchogram spreads segmentally in the dorsal part of both lungs. In addition, light ground-glass opacity is diffusely seen from the hilum. 


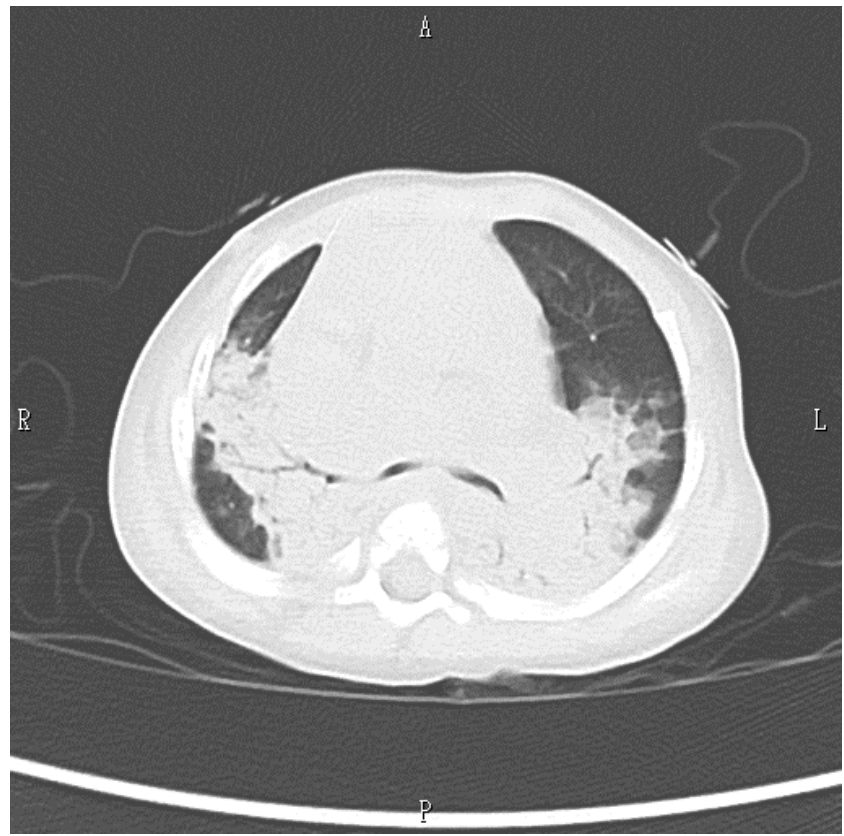

Figure 2. Case 3, Chest CT image on day 5 after onset. Ground-glass shadows or even shadows with bronchogram dominate the dorsal predominance of both lungs. Lung field consolidation reflects strong inflammation.

All cases of IgG/A/M levels were age-appropriate and normal ranges of $\mathrm{T} / \mathrm{B}$ and NK cell subsets were observed. Due to a serious deterioration of respiratory status in all cases, immunodeficiency tests for infection were performed and Pneumocystis jirovecii antigen, $\mathrm{HIV} \mathrm{Ab} / \mathrm{Ag}$, and $\beta$-D-glucan were negative. Antibody measurement with preserved serum demonstrated no co-infection with cytomegalovirus, Legionella, Chlamydia, or Mycoplasma. Echocardiographic findings and blood tests indicated no acute left ventricular cardiac dysfunction or fluid overload upon Pediatrics Intensive Care Unit (PICU) admission. For all cases, viral antigen was detected using a rapid diagnosis kit with a nasal aspiration on the day of admission. In addition, viral gene detection was performed using a one-step RT-PCR standard protocol in Cases 1 and 3. We diagnosed all patients with severe acute respiratory distress syndrome (ARDS) due to RS on the basis of no left heart failure, a $\mathrm{PaO}_{2} / \mathrm{FiO}_{2}$ ratio less than 200, and bilateral lung infiltration. Invasive mechanical ventilator management was performed with the aim of improving respiratory support and oxygenation with lung protection after admission. The effectiveness of steroid treatment for ARDS is controversial, depending on when it is started and the amount used. Because of a rapid respiratory deterioration including poor oxygenation, steroid pulse therapy consisting of methylprednisolone $30 \mathrm{mg} / \mathrm{kg}$ body weight was continuously administered for 3 days, and then post-treatment with $2 \mathrm{mg} / \mathrm{kg}$ was used for 4 days for a total of 7 days as one course. Case 2 post-treatment was extended by one week, and Case 3 was given two courses of steroid therapy. In all cases, steroid pulse therapy was considered to have been highly effective. Clinical symptoms and most laboratory markers improved, even when the clini- 
cal course was severe at admission, and returned to normal levels before discharge.

SP-D is mainly synthesized by type II pneumocytes and is an important component of innate pulmonary immunity. Sato et al. reported that adult ARDS patients who died had higher KL-6 levels than survivors [17]. SP-D reflectability of the condition is considered to be generalizable to adults and pediatric ARDS general [18]-[23]. Changes in SP-D and KL-6 levels before and after steroid treatment were marked, and altered SP-D levels suggested sensitive changes in the interstitial damage of the alveolar epithelial lining, including type II alveolar epithelial cells. This is because changes in SP-D levels paralleled changes in the $\mathrm{PaO}_{2} / \mathrm{FiO}_{2}$ ratio without any time lag in all cases. KL- 6 levels continued to increase for several days, even after the recovery of oxygenation following steroid treatment. The hypothesis that there is a time lag in serum values between KL-6 and SP-D in infants with ARDS by RS is considered as follows. First, this might be related to differences in the molecular size of SP-D and KL-6. SP-D has a molecular weight of about $500 \mathrm{kDa}$, whereas KL- 6 is much larger, $>5000$ $\mathrm{kDa}$ [24]. SP-D, which has a small molecular size, moves easily from the damaged tissue to the circulating blood, and therefore might better reflect tissue damage without a time lag. Second, the physiological environment for each factor is different. SP-D is a secreted protein and KL-6 is a cell membrane protein [25]. KL-6 can only enter the blood when it is cleaved from the cell membrane via a protease activated by inflammation, suggesting its entry to the blood is slightly delayed. Third, KL- 6 and SP-D are produced by type II alveolar epithelial cells; however, KL-6 is also produced by bronchial glands and SP-D is also produced by Clara cells. Differences in KL-6 and SP-D changes may also be related to the number of Clara cells and bronchial glands present. For these reasons, we considered SP-D levels were more sensitive to the pathological conditions than KL-6 levels. SP-D might be a biomarker to evaluate the effect of steroid pulse therapy and associated improvement in oxygenation for ARDS patients due to RS.

Kawasaki et al. reported that at the time of discharge, SP-D levels in infants with acute bronchiolitis due to RS did not recover to normal levels and were similar to those at admission [22]. SP-D levels were maintained until the time of discharge suggesting acute bronchiolitis due to RS continued to promote a partial inflammatory response even during the recovery period. In our cases, SP-D levels decreased to normal after steroid pulse therapy, even in severe ARDS cases, suggesting steroid pulse therapy reduced the inflammation of tissues and cells in which SP-D was present.

No previous reports have evaluated changes in the biomarkers SP-D during the clinical course of infants with ARDS due to RS who were successfully treated with steroid pulse therapy.

\section{Human Ethics}

Written Informed consent obtained from all patients' parents. 


\section{Authors' Countribution}

D. T. contributed to diagnosis management; S. I., T. O., and A. O. also contributed to treatment management; T. Y. provided expert clinical opinion; D. T. wrote the manuscript; S. I., T. O. A. O. and T. Y. clinically reviewed the manuscript and gave technical support. All authors read and approved the final manuscript.

\section{All Sources of Support}

Not applicable.

\section{Conflicts of Interest}

The authors declare no conflict of interest.

\section{References}

[1] Stein, R.T., Bont, L.J., Zar, H., Fernando, P., Polack, F.P., et al. (2017) Respiratory Syncytial Virus Hospitalization and Mortality: Systematic Review and Meta-Analysis. Pediatric Pulmonology, 52, 556-569. https://doi.org/10.1002/ppul.23570

[2] Gleezen, W.P. and Denny, F.W. (1973) Epidemiology of Acute Lower Respiratory Disease in Children. New England Journal of Medicine, 288, 498-505. https://doi.org/10.1056/NEJM197303082881005

[3] Hall, C.B., Simőes, E.A.F. and Anderson, L.J. (2013) Clinical and Epidemiologic Features of Respiratory Syncytial Virus. Current Topics in Microbiology and Immunology, 372, 39-57. https://doi.org/10.1007/978-3-642-38919-1_2

[4] Meert, K., Heidemann, S., Lieh-Lai, M., et al. (1989) Clinical Haracteristics of Respiratory Syncytial Virus Infections in Healthy versus Previous Compromised Hosts. Pediatric Pulmonology, 7, 167-170. https://doi.org/10.1002/ppul.1950070309

[5] Doucette, A., Jiang, X., Fryzek, J., Coalson, J., et al. (2016) Trends in Respiratory Syncytial Virus and Bronchiolitis Hospitalization Rates in High-Risk Infants in a United States Nationally Representative Database, 1997-2012. PLoS ONE, 11, e0152208. https://doi.org/10.1371/journal.pone.0152208

[6] Krasinksi, K. (1985) Severe Respiratory Syncytial Virus Infection; Clinical Features, Nosocomial Acquisition and Outcome. The Pediatric Infectious Disease Journal, 4, 250-257. https://doi.org/10.1097/00006454-198505000-00009

[7] Moler, F.W., Khan, A.S., Meliones, J.N., et al. (1992) Respiratory Syncytial Virus Morbidity and Mortality Estimates in Congenital Heart Disease Patients; a Recent Experience. Critical Care Medicine, 20, 1406-1413. https://doi.org/10.1097/00003246-199210000-00008

[8] Stretton, M., Ajizian, S.J., Mitchell, I., et al. (1992) Intensive Care and Outcome of Patients Infected with Respiratory Syncytial Virus. Pediatric Pulmonology, 13, 143-150. https://doi.org/10.1002/ppul.1950130304

[9] Navas, L., Wang, E., De Carvalho, V., et al. (1992) Improved Outcome of Respiratory Syncytial Virus Infections in a High-Risk Hospitalized Population of Canadian Children. The Journal of Pediatrics, 121, 348-354. https://doi.org/10.1016/S0022-3476(05)90000-0

[10] Cağlar Odek, C., Kendirli, T., Yaman, A., Aldemir-Kocabaş, B., et al. (2013) A Life-Threatening Respiratory Syncytial Virus Infection: A Previously Healthy Infant 
with Bilateral Spontaneous Pneumothorax and Acute Respiratory Distress Syndrome. The Turkish Journal of Pediatrics, 55, 539-542.

[11] Ravindranath, T.M., Gomez, A., Harwayne-Gidansky, I., et al. (2018) Pediatric Acute Respiratory Distress Syndrome Associated with Human Metapneumovirus and Respiratory Syncytial Virus. Pediatric Pulmonology, 53, 929-935.

https://doi.org/10.1002/ppul.24044

[12] Schene, K.M., van den Berg, E., Wösten-van Asperen, R.M., et al. (2014) FiO2 Predicts Outcome in Infants with Respiratory Syncytial Virus-Induced Acute Respiratory Distress Syndrome. Pediatric Pulmonology, 49, 1138-1144. https://doi.org/10.1002/ppul.22974

[13] Bernard, G.R., Arigas, A., Brigham, K.L., et al. (1994) The American-European Consensus Conference on ARDS. Definition, Mechanisms, Relevant Outcomes, and Clinical Trial Coordination. American Journal of Respiratory and Critical Care Medicine, 149, 818-824. https://doi.org/10.1164/ajrccm.149.3.7509706

[14] Flori, H., Dahmer, M.K., Sapru, A., Quasney, M.W. and Pediatric Acute Lung Injury Consensus Conference Group (2015) Comorbidities and Assessment of Severity of Pediatric Acute Respiratory Distress Syndrome: Proceedings from the Pediatric Acute Lung Injury Consensus Conference. Pediatric Critical Care Medicine, 16, S41-S50. https://doi.org/10.1097/PCC.0000000000000430

[15] Schwingshackl, A., Kimura, D., Rovnaghi, C.R., et al. (2016) Regulation of Inflammatory Biomarkers by Intravenous Methylprednisolone in Pediatric ARDS Patients: Results from a Double-Blind, Placebo-Controlled Randomized Pilot Trial. Cytokine, 77, 63-71. https://doi.org/10.1016/j.cyto.2015.10.007

[16] Judith, J.W., Mark, J., Rehena, S., et al. (2019) Mortality in Pediatric Acute Respiratory Distress Syndrome: A Systematic Review and Meta-Analysis. Journal of Intensive Care Medicine, 34, 563-571. https://doi.org/10.1177/0885066617705109

[17] Sato, H., Callister, M.E.J., Mumby, S., et al. (2004) KL-6 Levels Are Elevated in Plasma from Patients with Acute Respiratory Distress Syndrome. European Respiratory Journal, 23, 142-145. https://doi.org/10.1183/09031936.03.00070303

[18] Park, J., Pabon, M., Choi, K., et al. (2017) Plasma Surfactant Protein-D as a Diagnostic Biomarker for Acute Respiratory Distress Syndrome: Validation in US and Korean Cohorts. BMC Pulmonary Medicine, 17, 204. https://doi.org/10.1186/s12890-017-0532-1

[19] García-Laorden, M.I., Lorente, A.J., Flores, C., et al. (2017) Biomarkers for the Acute Respiratory Distress Syndrome: How to Make the Diagnosis More Precise. Annals of Translational Medicine, 5, 283. https://doi.org/10.21037/atm.2017.06.49

[20] Endo, S., Sato, N., Nakae, H., et al. (2002) Surfactant Protein A and D (SP-A, AP-D) Levels in Patients with Septic ARDS. Research Communications in Molecular Pathology and Pharmacology, 111, 245-251.

[21] Eisner, M.D., Parsons, P., Matthay, M.A., et al. (2003) Plasma Surfactant Protein Levels and Clinical Outcomes in Patients with Acute Lung Injury. Thorax, 58, 983-988. https://doi.org/10.1136/thorax.58.11.983

[22] Kawasaki, Y., Endo, K., Suyama, K., et al. (2011) Serum SP-D Levels as a Biomarker of Lung Injury in Respiratory Syncytial Virus Bronchiolitis. Pediatric Pulmonology, 46, 18-22. https://doi.org/10.1002/ppul.21270

[23] Kawasaki, Y., Aoyagi, Y., Abe, Y., et al. (2009) Serum KL-6 Levels as a Biomarker of Lung Injury in Respiratory Syncytial Virus Bronchiolitis. Journal of Medical Virology, 81, 2104-2108. https://doi.org/10.1002/jmv.21634 
[24] Wright, J.R. (1997) Immunomodulatory Functions of Surfactant. Physiological Reviews, 77, 931-962. https://doi.org/10.1152/physrev.1997.77.4.931

[25] Hilkens, J., Ligtengerg, M.J.L., Vos, H.L., et al. (1992) Cell Membrane-Associated Mucins and Their Adhesion-Modulating Property. Trends in Biochemical Sciences, 17, 359-363. https://doi.org/10.1016/0968-0004(92)90315-Z 